\title{
NOTE ON AN AMBIGUOUS CASE OF APPROXIMATION*
}

B)

\author{
DUNHAM JACKSON
}

1. Introduction. Let $f(x)$ be a continuous function of period $2 \pi$. The writer has discussed to some extent the existence and properties of a trigonometric sum $T_{m n}(x)$, of order $n$ at most, determined by the condition that the integral

$$
\int_{0}^{2 \pi}\left|f(x)-T_{m n}(x)\right|^{m} d x
$$

shall have the smallest possible value, $n$ being a given positive integer (or zero) and $m$ a given number $\geqq 1$, not necessarily integral.t The purpose of the present note is to inquire what becomes of the problem for values of $m$ less than 1.

For $m<0$, it is clear that the value of (1) can be brought arbitrarily near to zero by taking a sufficiently large constant for $T_{m n}(x)$, but can not be made equal to zero, so that the problem has no significance. For $m=0$, the integral (1) is always equal to $2 \pi$, unless there is a trigonometric sum which is identically equal to $f(x)$ throughout a point set of measure different from zero, in which case the integral is not defined. Attention may therefore be restricted to values of $m$ between 0 and 1 .

2. Existence of an approximating function. Let $m$ be a number of the interval $0<m<1$. When $m$ and $n$ are given, the value of the integral (1) has a lower limit $\gamma_{m n}$, which is positive or zero. It is to be inquired first whether there exists a trigonometric sum $T_{m n}(x)$ for which the lower limit $\gamma_{m n}$ is actually attained. The proof of existence given in the paper A for $m>1$, and in B for $m=1$, does not apply when $m<1$. It is possible nevertheless

* Presented to the Society, December 30, 1920.

$\dagger$ See D. Jackson, On functions of closest approximation, these 'Iransactions, vol. 22 (1921), pp. 117-128; Note on a class of polynomials of approximation, these Transactions, vol. 22 (1921), pp. 320-326; On the convergence of certain trigonometric and polynomial approximations, these Transactions, vol. 22 (1921), pp. 158-166.

These papers will be referred to by the letters $A, B, C$, respectively. 
to give a proof which is valid for any value of $m>0$, and which is as general in its scope as the earlier proofs mentioned, relating to a general class of approximating functions, of which trigonometric sums are only a very special case.

Let

$$
p_{1}(x), p_{2}(x), \ldots, p_{n}(x)
$$

be $n$ functions of $x$, continuous and linearly independent in the interval $a \leqq x \leqq b$. Let

$$
\varphi(x)=c_{1} p_{1}(x)+c_{2} p_{2}(x)+\cdots+c_{n} p_{n}(x)
$$

be an arbitrary linear combination of these functions with constant coefficients. The function $\varphi(x)$, then, might in particular be a trigonometric sum of order $n$, the interval being $(0,2 \pi)$; the number of term: would of course be $2 n+1$, instead of $n$, but this is a mere matter of notation, and is of no consequence. Let $H$ be the maximum of $|\varphi(x)|$ in $(a, b)$.

We shall assume, without repeating the proof here, a lemma of Sibirani,* to the effect that there exists a constant $P$, completely determined by the set of functions $p_{1}(x), \ldots, p_{n}(x)$, such that

$$
c_{k} \mid \leq P H \quad(k=1,2, \ldots, n)
$$

for all functions $\varphi(x)$.

Because of the uniform continuity of the $p$ 's, there will exist a number $d>0$, such that

$$
\left|p_{k}\left(x^{\prime}\right)-p_{k}\left(x^{\prime \prime}\right)\right| \leqq \frac{1}{2 n P} \quad(k=1,2, \ldots, n)
$$

whenever

$$
\left|x^{\prime}-x^{\prime \prime}\right| \leqq d
$$

this $d$ can be chosen once for all when the $p$ 's are given, since $n$ and $P$ are then determined, and it may be assumed that $d<\frac{1}{2}(b-a)$.

Let $\varphi(x)$ be any particular function of the form (2), and let $x_{0}$ be a point where $\mid \varphi$ ! takes on its maximum value $H$. Then, if

$$
\left|x-x_{0}\right| \leqq d
$$

\footnotetext{
* For a simple proof (with slightly different notation) see the paper A, Lemma I.
} 
it will follow from (3) and (4) that

$$
\begin{aligned}
\left|c_{k} p_{k}(x)-c_{k} p_{k}\left(x_{0}\right)\right| & \leqq \frac{H}{2 n} \quad(k=1,2, \ldots, n), \\
\left|\varphi(x)-\varphi\left(x_{0}\right)\right| & \leqq \frac{H}{2}, \\
|\varphi(x)| & \geqq \frac{H}{2} .
\end{aligned}
$$

The last relation holds throughout an interval of length at least $d$, since the points of the interval (5) are in $(a, b)$, on one side of $x_{0}$ at least, and hence, for $m>0$,

$$
G_{m b}=\int_{a}^{b}|\varphi(x)|^{m} d x \geqq d\left(\frac{H}{2}\right)^{m} .
$$

Consequently

$$
\begin{aligned}
& H \leqq 2\left(\frac{G_{m}}{d}\right)^{\frac{1}{m}}, \\
&\left|c_{k}\right| \leqq 2 P\left(\frac{G_{m}}{d}\right)^{\frac{1}{m}} \quad(k=1,2, \ldots, n) .
\end{aligned}
$$

That is,

There exists a constant $Q_{m}$, depending only on the p's and on the exponent $m$, such that

$$
\left|c_{k}\right| \leqq Q_{m} G_{m}^{\frac{1}{m}} \quad(k=1,2, \ldots, n)
$$

for all functions $\varphi(x)$; this is true for any value of $m>0$.

Let $f(x)$ be an arbitrary continuous function in $(a, b)$, and let

$$
g_{m}=\int_{a}^{b}|f(x)-\varphi(x)|^{m} d x
$$

When $m, f(x)$, and the $p$ 's are given, the value of $g_{m}$ will have a lower limit, positive or zero, for all possible functions $\varphi$, and it is to be shown that there will exist at least one $\varphi$ for which the lower limit is attained.

If $f(x)$ is linearly dependent on the $p$ 's, $\varphi$ can be made identically equal to $f$, and $g_{m}$ will thereby be made equal to its lower limit, zero. 
If $f(x)$ is not linearly dependent on the $p$ 's, $f$ and the $p$ 's together form a set of $n+1$ linearly independent functions. There will exist a constant $q_{m}$, depending only on $f$, the $p$ 's, and $m$, such that

$$
\left|c_{k}\right| \leqq q_{m} g_{m}^{\frac{1}{m}} \quad(k=1,2, \ldots, n)
$$

for all functions $\varphi$. So all the coefficients $c_{k}$ in any $\varphi$ which brings $g_{m}$ near its lower limit will belong to a bounded region, which may be regarded as closed, in the space of $n$ dimensions of which the $c$ 's are coördinates, and when $g_{m}$ is regarded as a function of the $c$ 's in this closed region, its lower limit will be a minimum which is actually attained for a suitable choice of the $c$ 's. In any case, then,

There will be at least one function $\varphi(x)$ for which the value of $g_{m}$ is a minimum.

3. Non-uniqueness of the approximating function. When $m>1$, it can be shown that the minimizing function $\varphi(x)$ is unique. ${ }^{*}$ The proof can be carried through also for $m=1$, provided that the functions $p_{k}(x)$ are suitably specialized. + It is readily seen that the corresponding assertion is not true in general for $m<1$, even if the discussion is restricted to functions $\varphi(x)$ which are finite trigonometric sums.

For example, let $\varepsilon$ be a small positive quantity; let

$$
\begin{aligned}
& f(x)=1, \quad \frac{\pi}{2}+\varepsilon \leqq x \leqq \frac{3 \pi}{2}-\epsilon \\
& f(x)=-1, \quad 0 \leqq x \leqq \frac{\pi}{2}-\varepsilon, \quad \frac{3 \pi}{2}+\varepsilon \leqq x \leqq 2 \pi
\end{aligned}
$$

and let $f(x)$ be linear and continuous from $(\pi / 2)-\varepsilon$ to $(\pi / 2)+\varepsilon$ and from $(3 \pi / 2)-\varepsilon$ to $(3 \pi / 2)+\varepsilon$, and of period $2 \pi$ for all values of $x$. That is, as nearly as is consistent with continuity, $f(x)$ is equal to +1 throughout half a period, and to -1 throughout the other half. Let $\varphi(x)$ in this case be a finite trigonometric sum of order zero, that is, a constant, and let $m=\frac{1}{2}$. Then it is clear, from the symmetry of the definition of $f(x)$, that if $g_{m}$ takes on its minimum value for $\varphi=c_{0}$, it will take on the same value for $\varphi=-c_{0}$, and so there will be two different minimizing constants, unless $c_{0}=0$; but the value of $g_{m}$ for $\varphi=0$ can be made arbitrarily near to $2 \pi$, and its value

* See $A, \S 6$.

$\dagger$ See $B, \S \S 3,4$. The proof is written out for the polynomial case, but the method applies equally well to the problem of approximation by trigonometric sums; cf. B, end of $\S 1$. 
for $\varphi= \pm 1$ arbitrarily near to $\pi \sqrt{\overline{2}}$, by taking $\varepsilon$ sufficiently small, so that there are cases in which $\varphi=0$ certainly does not give the minimum. For any other value of $m$ between 0 and 1, the approximate values of $g_{m}$ for $\varphi=0$ and for $\varphi= \pm 1$ would be $2 \pi$ and $2^{m} \pi$ respectively, and the conclusion would be the same. A similar example could be given for the case of polynomial approximation.

4. Convergence. In view of the failure of the property of uniqueness, it is perhaps all the more remarkable that the question of the convergence of the approximating functions toward the value $f(x)$, when $m$ is held fast and $n$ is allowed to become infinite, can be treated, in the special case of trigonometric approximation, in almost exactly the same way as for $m \geq 1$. It is sufficient to refer to the discussion in the paper $C$, with the remark that the only property of the minimizing function used explicitly is that it gives the integral at least as small a value as a specified function of the same form. Let a trigonometric sum of order $n$ or lower which gives the integral its minimum value, for given $m$ and $n$, be referred to as an approximating function (no longer the approximating function) of order $n$, corresponding to the exponent $m$; then the reasoning of $\mathrm{C}$ will lead to the conclusion:

If $f(x)$ is a continuous function of period $2 \pi$ which can be represented by " trigonometric sum of order $n$ or lower with an error not exceeding $\varepsilon_{n}$, if $T_{m n}(x)$ is an approximating function of order $n$ for $f(x)$, corresponding to the exponent $m$, and if $\varrho_{n}$ is the maximum of $\left|f(x)-T_{m n}(x)\right|$, then

$$
\varrho_{n} \leqq l_{m} n^{\frac{1}{m}} \varepsilon_{n}
$$

where $l_{m}\left(=k_{m}+1\right.$ of the paper ( $)$ is a constant depending only on $m$.

The deduction of theorems on convergence follows as in $\mathrm{C}$, on the basis of the general theorems on trigonometric approximation there referred to. ${ }^{*}$ If there are two or more approximating functions for a given value of $n$, it will be immaterial for the truth of the conclusion which is chosen. There will be occasion now to assume that $f(x)$ has a derivative, of the first or of higher order, possessing a certain degree of continuity; the results need not be set down in further detail.

The discussion of convergence can be extended to values of $m<1$ in the polynomial case as well. $t$

" See D. Jackson, On the approximate representation of an indefinite integral, etc., these Transactions, vol. 14 (1913), pp. 343364.

$\dagger$ Cf. the paper $\mathrm{C}, 8 \S 5,6$.

UNIVERSITY OF MINNESOTA.

MinNeAPOLIS, MinN. 